\title{
Caractéristiques et spécificités du karst de la Montagne de Reims ; comparaison avec les autres karsts de contact lithostratigraphique de l'est du Bassin parisien
}

Characteristics and specificities of the karst of the Montagne de Reims;

comparison with other of lithostratigraphic contact karsts of the Eastern Paris Basin

Eigenschaften und Besonderheiten des Karsts der Montagne de Reims - Vergleich mit den anderen von lithostratigraphischen Kontakt Karsten des östlichen Pariser Beckens

Olivier Lejeune et Alain Devos

\section{OpenEdition} Journals

Édition électronique

URL : http://journals.openedition.org/rge/3933

DOI : 10.4000/rge.3933

ISSN : 2108-6478

Éditeur

Association des géographes de l'Est

Édition imprimée

Date de publication : 1 septembre 2001

ISSN : 0035-3213

Référence électronique

Olivier Lejeune et Alain Devos, « Caractéristiques et spécificités du karst de la Montagne de Reims ; comparaison avec les autres karsts de contact lithostratigraphique de l'est du Bassin parisien », Revue Géographique de l'Est [En ligne], vol. 41 / 4 | 2001, mis en ligne le 22 juillet 2013, consulté le 08 septembre 2020. URL : http://journals.openedition.org/rge/3933 ; DOI : https://doi.org/10.4000/rge. 3933

Ce document a été généré automatiquement le 8 septembre 2020

Tous droits réservés 


\section{Caractéristiques et spécificités du karst de la Montagne de Reims ; comparaison avec les autres karsts de contact lithostratigraphique de l'est du Bassin parisien}

Characteristics and specificities of the karst of the Montagne de Reims; comparison with other of lithostratigraphic contact karsts of the Eastern Paris Basin

Eigenschaften und Besonderheiten des Karsts der Montagne de Reims - Vergleich mit den anderen von lithostratigraphischen Kontakt Karsten des östlichen

Pariser Beckens

Olivier Lejeune et Alain Devos

\section{Introduction}

1 Les karsts de contact lithostratigraphique de l'est de la France ont été récemment étudiés dans le cadre de recherches universitaires (Rodet, 1992; Gamez, 1995 ; Jaillet, 2000). Celui de la Montagne de Reims a fait l'objet d'études locales (Guérin 1959, Dumont, 1959), biospéléologiques (Chauvin, 1985, 1986) et endokarstique (Rodet, 1992) qui n'ont pas traité de sa dynamique et de son organisation spatiale. L'article rappelle le contexte morphologique régional du karst de la Montagne de Reims, puis analyse sa dynamique. Une comparaison avec les autres karsts de contact lithostratigraphique de l'est de la France met en évidence ses spécificités. 


\section{Le contexte régional et le karst de la Montagne de Reims}

\section{A. La montagne de Reims et la cuesta d'Ile-de-France}

2 La Montagne de Reims, correspondant au revers (altitude maximale de $283 \mathrm{~m}$ ) de la cuesta d'Ile-de-France, décrit le saillant le plus oriental de cette cuesta entre les deux entonnoirs de percée cataclinale de la Vesle au nord et de la Marne au sud (fig. 1). Elle domine de plus de $150 \mathrm{~m}$ le plateau de revers crayeux de la cuesta de Champagne. Les limites de la Montagne de Reims sont, à l'est, au nord et au sud le front de cuesta. À l'ouest, celle-ci est limitée par la vallée de la Germaine.

Figure 1 : Carte synthétique des principaux karsts de contact lithostratigraphique dans la partie Nord-Est du Bassin parisien

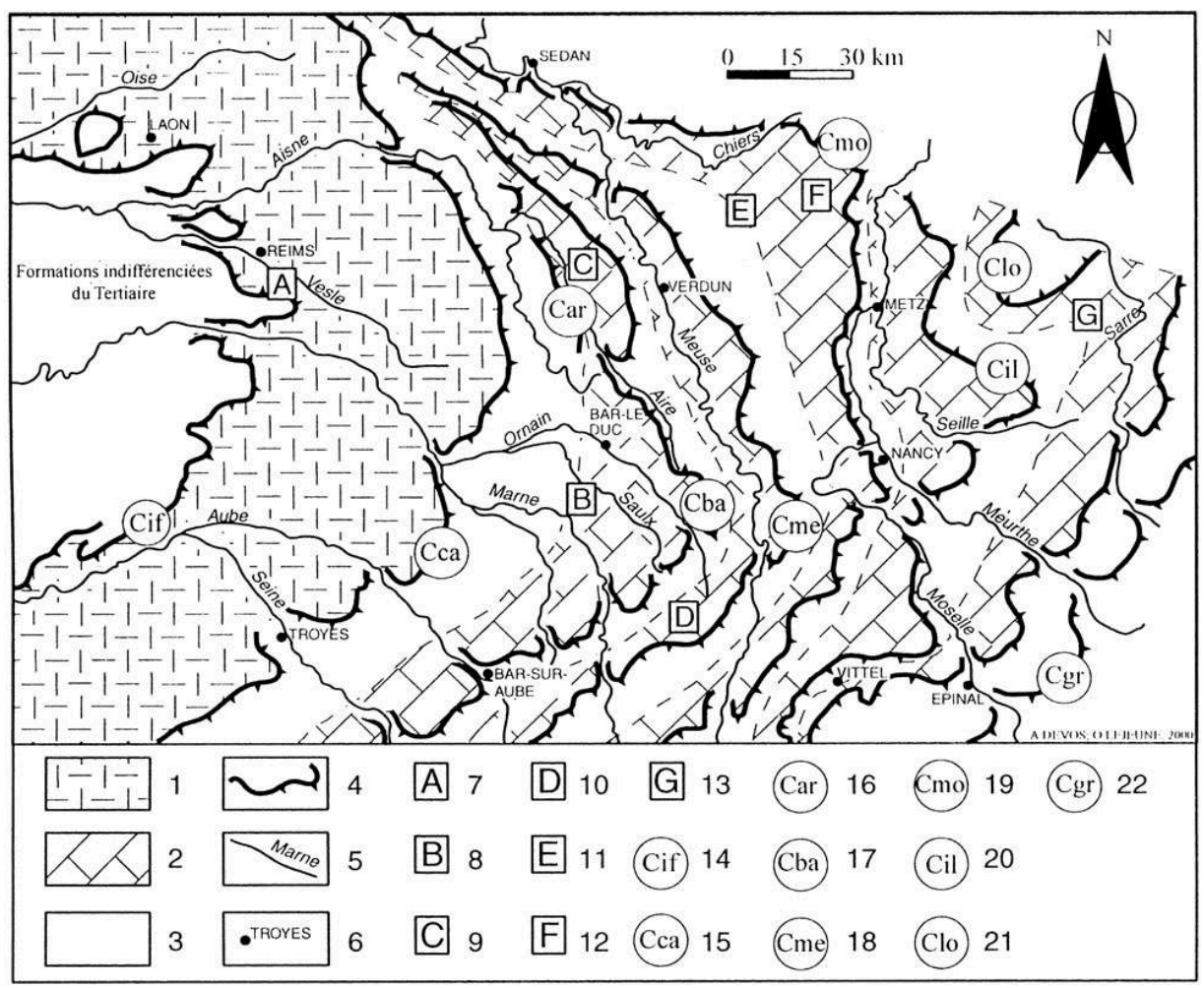

1 : Craie, 2 : Calcaires cohérents, 3 : Autres roches indifférenciées, 4 : Front de cuesta, 5 : Cours d'eau, 6 : Localité repère, $7:$ Karst de la Montagne de Reims, 8 : Karst du Barrois, 9 : Karst de l'Argonne, 10 : Karst du Haut-Pays, 11 : Karst de la Woëvre septentrionale, 12 : Karst du Pays-Haut, $13:$ Karst du Muschelkalk, 14 : Côte de l'lle-de-France (Tertiaire/Campanien), 15 : Côte de Champagne (Turonien/ Coniacien), 16 : Côte d'Argonne (Albien/Cénomanien), 17 : Côte du Barrois (Porlandien/Kimmeridgien), 18 : Côte de Meuse (Oxfordien/Callovien), 19 : Côte de Moselle (Bajocien/Toarcien), 20 : Côte Infraliasique, 21 : Côte du Wardnt (Muschelkakl), 22 : Côte des Grès (Buntsanstein).

La Montagne de Reims forme ainsi l'interfluve Marne-Vesle incisé par les affluents de rive droite de la Marne (Germaine, Livre) conquérants par rapport à ceux de la Vesle. Ces cours d'eau découpent la Montagne de Reims en trois lambeaux de plateaux, de Saint-Imoges à l'ouest, de Ville-en-Selve au centre et de Saint-Basle à l'est (fig. 4).

Son front de côte s'inscrit dans les formations éocènes et paléocènes (base du Tertiaire) surmontant la craie campanienne (Crétacé terminal) (fig. 2). La partie sommitale de la 
craie est affectée par une surface d'altération réalisée pendant une lacune de sédimentation (Dano-Montien). Les roches tertiaires sont principalement représentées par des sables (Thanétien et Cuisien), des argiles (Sparnacien) et des marnes (Lutétien et Ludien). Localement, les horizons calcaires du Bartonien arment le font de côte. Les argiles à meulières du Sannoisien tapissent la surface substructurale de la Montagne de Reims. Elles sont constituées de blocs massifs et compacts de meulières inclus dans une masse d'argiles. Elles témoignent d'une altération superficielle en milieu continental (Laurain, 1980, 1981 ; Guérin, 1985).

Figure 2 : Log lithostratigraphique de la Montagne de Reims au niveau de Verzenay-Verzy

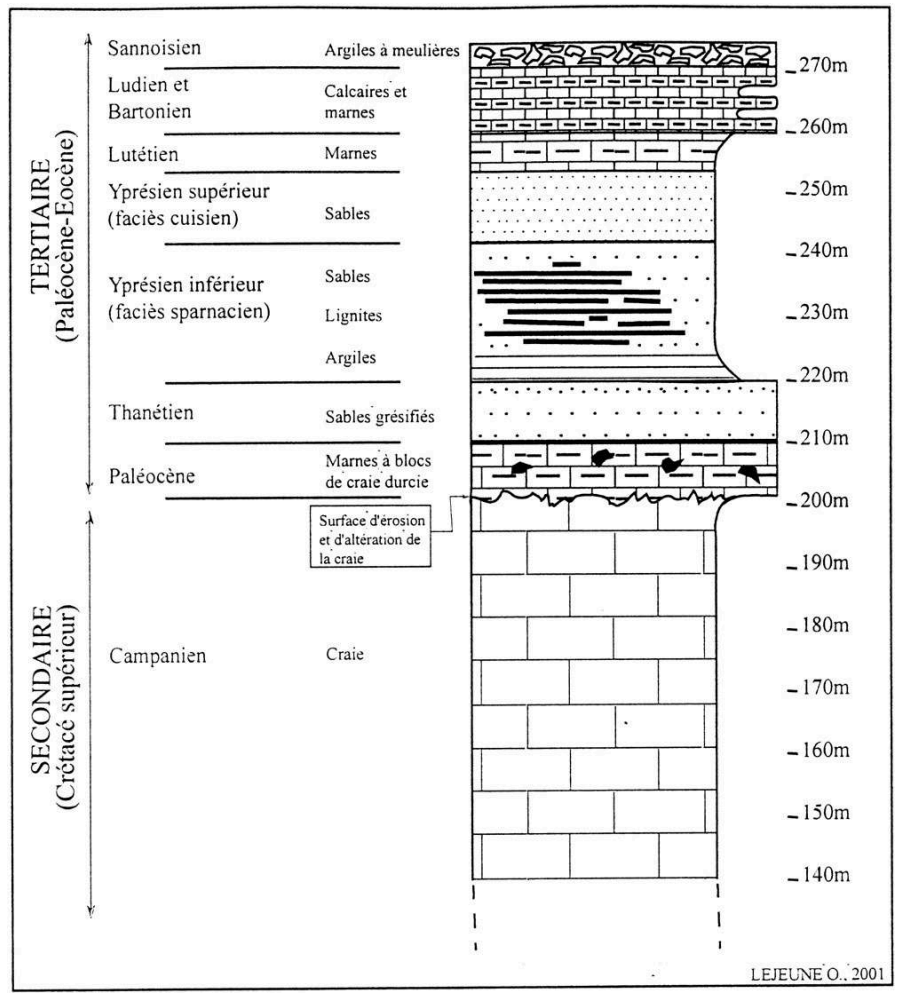

5 Cette cuesta présente ici certaines particularités par rapport aux autres cuestas de l'est de la France, de par son fort commandement $(180 \mathrm{~m})$, son gradient hydraulique et aussi par son profil à pente douce lié à une série de glacis partiellement entaillés par des vallons.

6 Son contexte morphostructural est également original: en effet, la lacune de sédimentation du Dano-Montien est contemporaine de la surface d'érosion et d'altération de la craie qui porte des blocs de craie marneuse. Les dépôts paléocènes et éocènes présentent une grande diversité de modes de sédimentation littorale marine, avec des apports détritiques continentaux (formations de mangrove, de dunes, de cordons de galets). La Montagne de Reims, en position anticlinale exondée lors de la sédimentation paléocène (fig. 3), sépare la mer thanétienne au nord d'un couloir littoral au sud (Schroeder, in Duprat, 1997) qui se termine par un cône de déjection (situé à Reims dans l'actuel entonnoir de percée cataclinale de la Vesle). L'éperon de Verzy correspond à l'ancien apex du cône, ce qui explique la prédominance des carbonates et la grésification par un ciment calcaire des sables thanétiens (Guillevin \& Renbard, 1975, in Duprat, 1997) provenant de l'ablation des reliefs crayeux 
environnants. Ces sables grésifiés déterminent des replats structuraux, dont la discontinuité s'explique par des changements latéraux et verticaux de faciès et la variation brutale des épaisseurs. Cette complexité géologique régionale contraste avec le mode de sédimentation homogène des cuestas du Jurassique.

Figure 3 : Situation de la Montagne de Reims lors de la sédimentation paléocène marine (Thanétien)

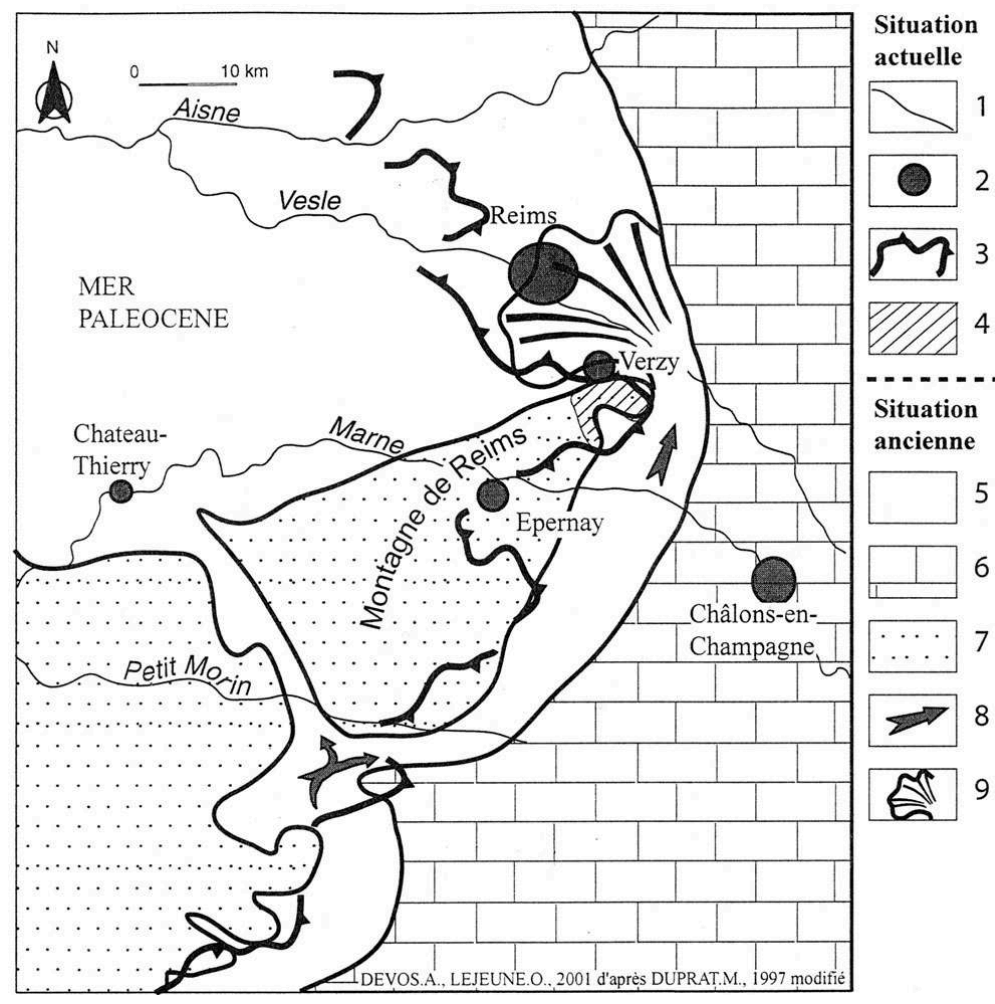

1 : Cours d'eau repère, 2 : Localité repère, 3 : Côte de l'lle-de-France, $4:$ Zone karstifiée actuellement, 5 : Paléocène marin (Thanétien), 6 : Relief de craie émergé, $7:$ Zone exondée, 8 : Sens des apports clastiques, 9 : Cône de déjection à dépôts clastiques à galets

Les formations éocènes et paléocènes constituent la quasi intégralité des affleurements de la côte au nord de la Vesle, où la dépression orthoclinale est entièrement dégagée dans les sables thanétiens non grésifiés. Par contre, en Montagne de Reims, elles constituent uniquement la partie extrême sommitale du front de côte, la dépression orthoclinale étant dégagée dans la craie en position d'amont pendage (Montagne de Reims orientale).

Enfin, la lithologie très variée entraîne des binômes de cohérence et de perméabilité sur le front de côte. Ces derniers déterminent des aquifères, dont le plus important est celui des sables cuisiens sur les argiles sparnaciennes. Cette nappe entretient des secteurs humides alimentés par des émergences de débordement ou de trop plein audessus des replats structuraux du Thanétien.

\section{B. Le karst de la Montagne de Reims}

9 L'intégralité des phénomènes karstiques recensés à ce jour se concentre dans la partie orientale de la Montagne de Reims (fig. 4). 
Figure 4 : Karst de contact lithostratigraphique de la Montagne de Reims

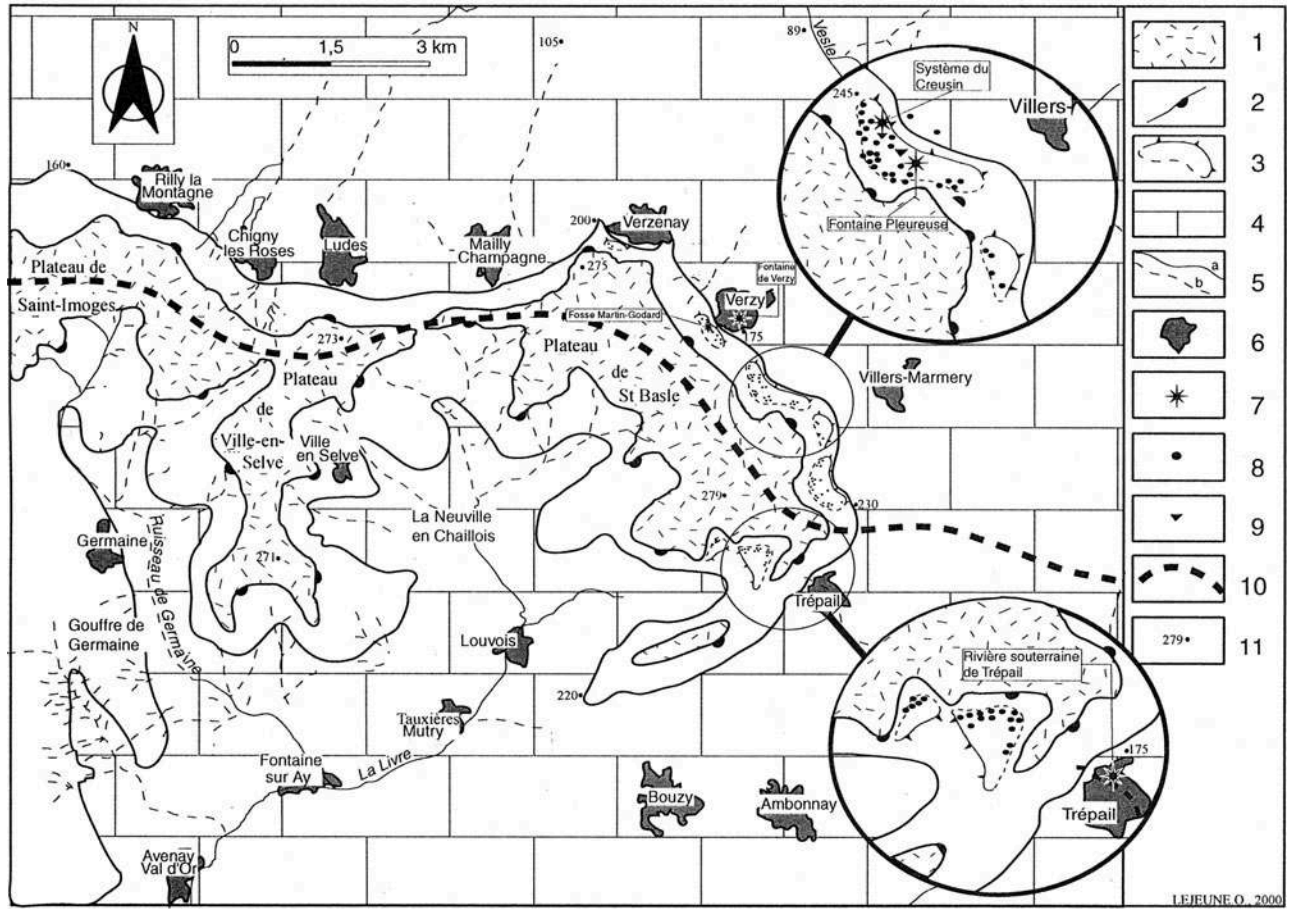

1 : Revers du plateau (surface structurale des argiles à meulières du Sannoisien), 2 : Talus de la cuesta de l'lle-de-France dans les roches tertiaires, 3 : Replat structural, 4 : Terrain crayeux campanien, 5 : cours d'eau (a) rivière pérenne, (b) rivière intermittente, 6 : Village, 7 : Réseau pénétrable, 8 : Doline, 9 : Perte remarquable, 10 : Ligne de partage des eaux (interfluve Marne/Vesle), 11 : Point coté en mètres

Les formes exokarstiques sont représentées par des dolines, des gouffres et de rares vallées aveugles. Elles s'alignent en liseré sur le replat structural des sables grésifiés, au pied d'un versant réglé et dégagé dans les formations éocènes. Les dolines sont de tailles diverses : de moins d'un mètre à plus de $50 \mathrm{~m}$ de diamètre, à fond plat ou en cuvette ( $40 \%$ ) et en entonnoir (60\%). On constate localement un étagement des dolines avec des phénomènes de recul de perte en fond de vallon. Les dolines inondées débordent pour former un écoulement vers les dolines inférieures. Les gouffres qui trépanent la couverture sableuse et la craie restent rares (fosse Martin Godard, gouffre du Creusin). Ils s'ouvrent en fond de doline mais restent de taille modeste.

11 Les réseaux endokarstiques (dans les sables grésifiés) dentritiques ou méandriformes à collecteur unique (Fontaine Pleureuse, Creusin) présentent un développement compris entre 100 et $300 \mathrm{~m}$, quasi horizontal, à faible dénivelée (moins de deux mètres), avec des affluents non pénétrables. Les galeries, à profil transversal ovoïde, de petite taille (1,5 $\mathrm{m}$ de diamètre) sont retouchées localement par l'homme (alimentation en eau potable).

Les réseaux de la craie sont mal connus. Les réseaux de la Grande Fontaine de Verzy et de Trépail sont les seuls pénétrables. Leurs développements respectifs sont de $560 \mathrm{~m}$ et $850 \mathrm{~m}$ (Rodet, 1985, 1992, 1997). Les réseaux calés sur diaclases et failles sont matures et présentent des siphons, des formes d'érosion torrentielle et des marques de captures successives.

13 Les facteurs explicatifs sont d'ordre géologique et hydrodynamique. En effet, les roches karstifiées sont les sables grésifiés du Thanétien et la craie campanienne. La 
grésification des sables thanétiens cimentés par une matrice calcaire inhérente au contexte de sédimentation paléocène détermine une série de replats structuraux karstifiés sur le front de côte. Le gradient hydraulique élevé, lié à la situation d'amont pendage, à l'extrémité orientale de la Montagne de Reims, entretient également une énergie potentielle plus importante dans la karstification (Quinif, 1998).

\section{Dynamique du karst de la Montagne de Reims}

\section{A. Dynamique des écoulements}

14 La principale dynamique des karsts de bas plateau n'est pas inhérente au gradient hydraulique trop faible, mais aux modalités de concentration des eaux de surface vers le karst. Ce sont donc les contrastes de perméabilité et de porosité qui sont déterminants: le binôme couche imperméable sur couche perméable détermine un liseré de pertes au contact lithostratigraphique (fig. 5a) alors que le binôme inverse (couche perméable sur couche imperméable) détermine un aquifère avec une ligne de sources.

15 L'Éocène, par sa diversité lithologique, détermine de nombreux aquifères multicouches, discontinus, perchés, mais d'intérêt local (niveaux calcaires du Lutétien au Ludien). La nappe des sables du Cuisien, dont le mur aquifère est constitué par les argiles sparnaciennes, est le principal réservoir du front de côte. Les sables grésifiés du Thanétien constituent également un aquifère discontinu et karstifié, calé sur le niveau des marnes à blocs de craie du Paléocène inférieur à $205 \mathrm{~m}$ d'altitude (fig. 5b). Cependant, le principal aquifère du front de côte est l'aquifère de la craie campanienne dont la surface piézométrique est située à $140 \mathrm{~m}$ d'altitude, soit à plus de $60 \mathrm{~m}$ endessous de la zone d'infiltration des sables grésifiés. 
Figure 5 : (a) Exemple d'un des replats structuraux (Lambert 1, x:733.8; y:1160.9, z:240) sur le versant nord de la Montagne de Reims et des phénomènes karstiques qui s'y développent. (b) Coupe schématique du système karstique étagé du Creusin dans les sables grésifiés thanétiens et dans la craie campanienne

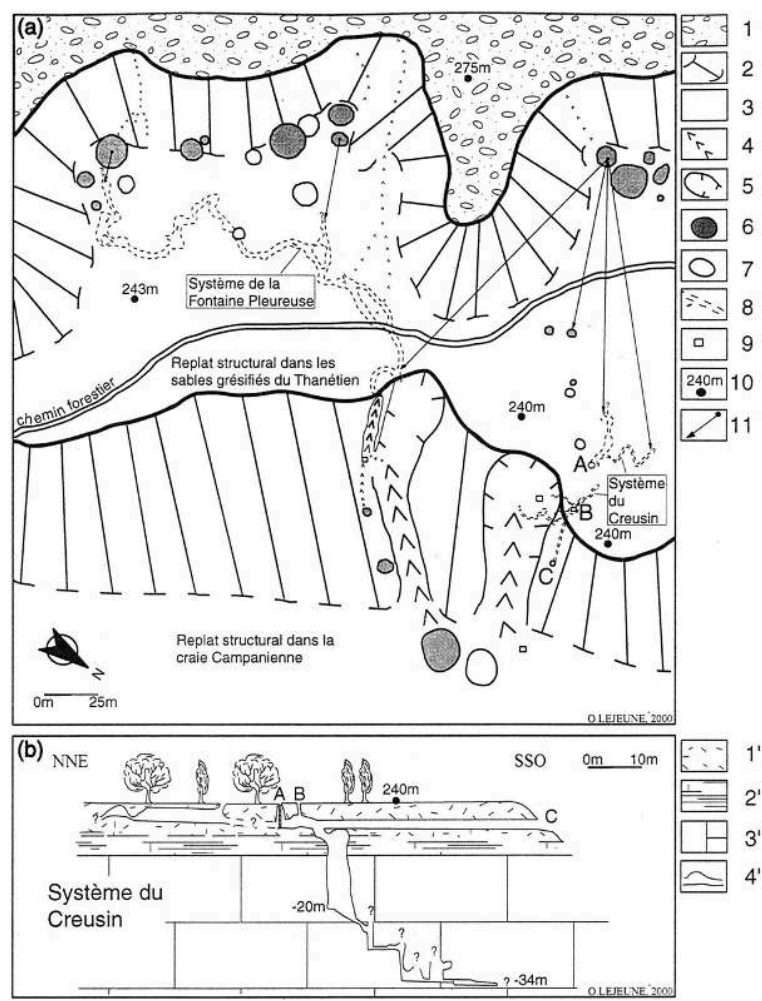

(a) 1 : Surface des argiles à meulières du Sannoisien (partie sommitale du plateau), 2 : Versant réglé, 3 : Replat structural (dans les sables grésifiés ou la craie), 4 : Petit vallon encaissé, 5 : Grand vallon, 6 : Doline en entonnoir, 7 : Doline en cuvette, 8 : Réseau souterrain, 9 : Regard anthropique sur un réseau souterrain, 10 : Point coté, 11 : Liaison prouvée par traçage ; (b) 1' : Sables du Thanétien, 2' : Marnes à blocs de craie du Paléocène inf, $3^{\prime}$ : Craie du Campanien, 4' : Réseau karstique

Les sources issues des nappes de l'Éocène constituent des écoulements bien hiérarchisés sur les niveaux argileux dans de petits vallons qui incisent les versants réglés du front de côte. Ces vallons festonnent le liseré karstique des sables grésifiés qui absorbent les écoulements. La couverture éocène joue ainsi le rôle de pourvoyeur et de concentration des eaux vers le karst des sables thanétiens. Par drainance, elle alimente également, telle une "compresse humide $»^{1}(\mathrm{Gamez}, 1995)$ l'aquifère de la craie. Lorsque les gouffres trépanent la couverture et la craie (Fosse Martin-Godard, fig. 4 et 6), ces écoulements de surface, concentrés et agressifs (pauvreté des horizons calcaires) alimentent rapidement et directement la zone d'infiltration de l'aquifère de la craie. Par contre, lorsque l'exokarst affecte uniquement les sables thanétiens, l'organisation des écoulements souterrains est plus complexe. En effet, les traçages effectués (Lejeune, 2000) témoignent de l'indépendance et de l'intensité du karst des sables grésifiés par rapport à celui de la craie. Les taux de restitution compris entre 85 et $92 \%$ mettent en évidence des écoulements concentrés et rapides (58 à $85 \mathrm{~m} / \mathrm{h}$ ). Mais, les écoulements, localement diffluents (multiplicité des points de sortie et aplatissement de la courbe de restitution), sont caractéristiques de milieux sableux moins cimentés. 
Figure 6 : (a) Mouvement de terrain en relation avec le karst au niveau de la Fosse Martin-Godard (Lambert 1, x: 733.2, y: 1161.77, $3: 224.3$ ) ; (b) Mouvement de terrain et Fosse Martin-Godard en coupe

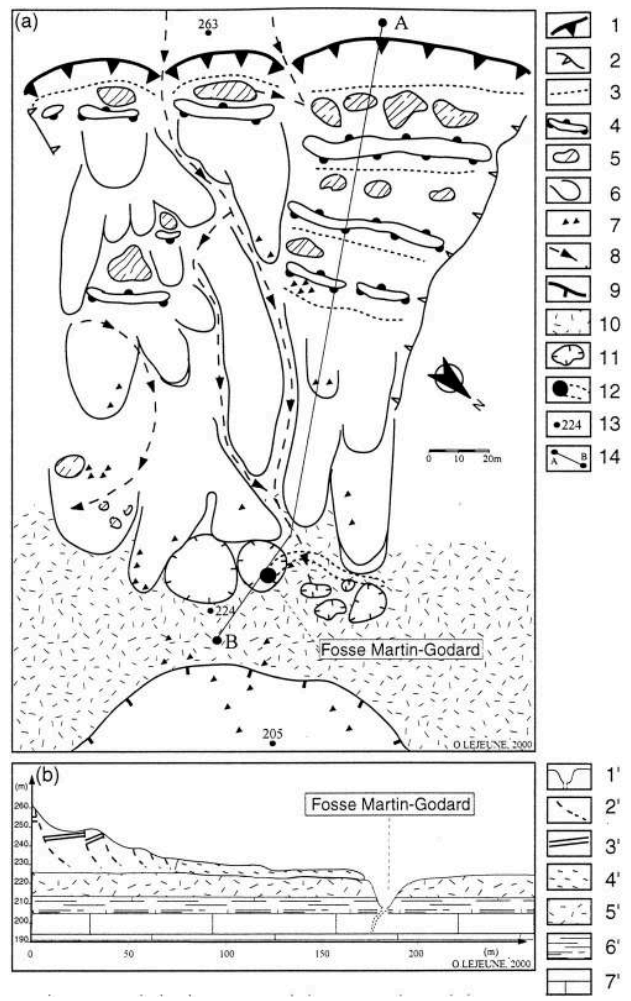

(a) 1 : Escarpement de tête de glissement, 2 : Escarpement latéral, 3 : Concavité, 4 : Masse soulevée, 5 : Mare due à une contre-pente, 6 : Limite de coulées, 7 : Bloc de meulières (sup à $5 \mathrm{~m}$ ), 8 : Ecoulement temporaire dans des vallons en « $\vee », 9$ : Rebord extérieur du replat des sables grésifiés, 10 : Replat des sables grésifiés, 11 : Doline, 12 : Gouffre et réseau souterrain, $13:$ Point coté, $14:$ Tracé de la coupe de la figure $5 \mathrm{~b}$; (b) $1^{\prime}$ : Doline et réseau souterrain, 2': Plan de glissement supposé, $3^{\prime}$ : Masse de roches meubles glissées du Tertiaire supérieur, $4^{\prime}$ : Coulées, $5^{\prime}$ : Sables grésifiés du Thanétien, $6^{\prime}$ : Marne à blocs de craie durcie du Paléocène inférieur, $7^{\prime}$ : Craie du Campanien

17 Des exsurgences jalonnent alors le rebord du replat structural des sables grésifiés et alimentent un réseau hydrographique qui se perd de manière diffuse quelques mètres plus bas sur la craie.

Cependant, localement (réseau du Creusin, fig. 5b), l'endokarst des sables grésifiés est trépané à la base par des gouffres aveugles du karst de la craie qui capturent ainsi les écoulements sus-jacents, aux dépens des exsurgences du rebord du replat structural. Dans ce cas, tel un épikarst, l'aquifère paléocène joue le double rôle de compresse humide et de concentration des écoulements vers la zone d'infiltration rapide de l'aquifère de la craie.

L'aquifère de la craie est ici tout aussi complexe, avec un endokarst et des exsurgences perchés entre 170 et $180 \mathrm{~m}$ d'altitude (Fontaine de Trépail, Grande Fontaine de Verzy), soit $40 \mathrm{~m}$ au-dessus de la zone saturée. Cet aquifère présente ainsi trois modes d'introduction des eaux, par des pertes diffuses sur la craie, par des pertes localisées (karst de contact lithostratigraphique) et par un karst étagé qui retarde, concentre ou diffuse les écoulements.

Ces caractéristiques permettent de définir le karst de la Montagne de Reims comme un « karst ternaire » (Jaillet, 2000). 
Cependant, si la zone d'infiltration de l'aquifère de la craie est complexe, la zone noyée ou saturée est caractéristique des milieux poreux et isotropes. Elle constitue le plus grand aquifère libre du bassin de Paris, tant par sa surface que par son volume, et contraste ainsi avec l'étroitesse de la zone karstifiée. Ces caractéristiques, ainsi que la situation d'aval pendage en limite de nappe captive sous couverture éocène, expliquent la pondération du régime hydrologique des sources et des cours d'eau inhérente à la capacité tampon du matériau crayeux. Cet aspect lisse considérablement, voire supprime l'influence du karst sur le comportement hydrodynamique des sources de fond de vallée.

\section{B. Dynamique des versants}

La morphodynamique de la côte d'Ile-de-France est marquée par des mouvements de terrain qui s'expliquent par des facteurs morphostratigraphiques, climatiques et hydrologiques favorables (Marre, 1987) : la faible cohésion des matériaux éocènes et paléocènes (argiles sparnaciennes, sables cuisiens, sables thanétiens non grésifiés); la pente (qui peut atteindre $45^{\circ}$ dans la partie sommitale du front de côte) et le dénivelé important $(180 \mathrm{~m})$; le contexte hydrogéologique favorable à la constitution de nappes d'eau en formations sableuses, perchées sur les argiles basales du Sparnacien; le « rôle lubrifiant» des montmorillonites et illites sparnaciennes (à la base du plan de glissement) humectées par les nappes sus-jacentes et les émergences de débordement.

Ces mouvements de terrain présents sur les versants de la Montagne de Reims sont de deux types: des glissements rotationnels et des coulées qui apparaissent ensemble ou séparément sur le versant. Ainsi, l'association classique "versant réglé - glacis ${ }^{2}$ de la côte (forme lisse) est détruite par ces phénomènes qui font apparaitre de haut en bas de nombreuses cicatrices d'arrachement, des replats à contre-pente (propices à la stagnation des eaux) et des coulées boueuses (fig. 6).

Les mouvements de terrain entretiennent une étroite relation avec la karstification (fig. 6) : d'une part, ils conditionnent la concentration et l'organisation des écoulements de surface vers l'exokarst, et d'autre part, ils jouent le rôle d'impluviums en forme d'entonnoir avec un bassin de réception correspondant à la tête du glissement et un chenal d'écoulement unique vers chaque perte dans la zone des coulées.

Ainsi les mouvements de terrain, par leur rôle de réception et de transit des eaux pluviales et souterraines, participent à la karstification. À l'inverse, les coulées boueuses tendent à obstruer l'exokarst et à stabiliser le versant.

En effet, le karst actif absorbe le matériel des coulées et participe à leur déstabilisation. L'eau ruisselle dans les ravines inscrites dans les matériaux tertiaires et se perd dans les formes exokarstiques qui jalonnent les replats des sables grésifiés. L'incision linéaire des hauts de versant se termine en vallées aveugles sur les sables grésifiés et est remplacée par un écoulement souterrain de type karstique. L'érosion devient ainsi souterraine (ablation karstique) et préserve la topographie de surface (immunité karstique). On peut donc penser que cette immunité karstique participe à la conservation de la partie orientale de la Montagne de Reims, alors que les percées cataclinales de la Vesle et de la Marne progressent vers l'aval, rapidement, sous l'effet des mouvements de terrain. 
On assiste donc à la concurrence de deux dynamiques, l'une karstique, l'autre de versant. La première tend à préserver les formes structurales (replats des sables grésifiés), alors que la seconde (mouvements de terrain) tend à les modifier.

\section{Le karst de la Montagne de Reims comparé aux autres karsts de l'est de la France}

Le karst de la Montagne de Reims a été comparé à d'autres karsts de l'est de la France dont, d'ouest en est, ceux du Barrois (Jaillet et Gamez, 1995 ; Jaillet, 2000), d'Argonne (Durup de Baleine, 1989, 1991), du Haut-Pays (Devos, 1996), de Wöevre septentrionale (Gamez, 1995), du Pays-Haut (Devos, 1991) et enfin du Wardnt (Frischmann, 1997). Un schéma typologique des différents sites de karst de l'est du Bassin parisien (fig. 7) a été dressé à partir d'observations de terrain et de recherches bibliographiques. Il présente non seulement le contexte morphologique des karsts lorrains mais aussi les modalités d'écoulement dans l'aquifère calcaire. Ce schéma permet de comparer le karst de la Montagne de Reims à d'autres karsts lithostratigraphiques de l'est de la France et de dégager ses caractères communs ainsi que ses spécificités.

Figure 7 : Schémas typologiques des différents sites de karst de l'est du Bassin parisien

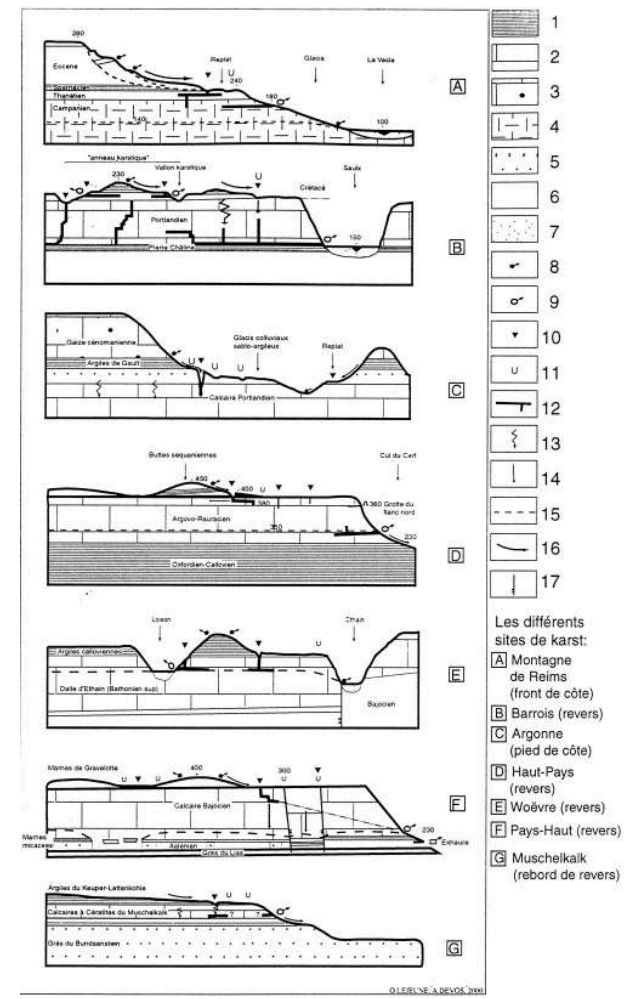

1 : Argile, 2 : Calcaire, 3 : Gaize, 4 : Craie, 5 : Sable, 6 : Autres roches, $7:$ Alluvions, 8 : Source, 9 : Source karstique, 10 : Gouffre, 11 : Doline, 12 : Réseau endokarstique, 13 : Drainance, 14 : Perte localisée, 15 : Surface piézométrique, 16 : Ruissellement, 17 : Faille 


\section{A. Caractères communs}

Reims est situé sur un contact lithostratigraphique caractéristique d'un binôme de perméabilité (Nicod, 1992,1994 et 1995). Les phénomènes exokarstiques affectent donc, non seulement la roche calcaire, mais également la couverture non carbonatée. Le rôle de cette dernière, imperméable ou semi-perméable, est fondamental dans la formation et l'évolution du karst (Jaillet, 2000). Elle conditionne la dynamique du karst par sa nature (ruissellement sur argiles, constitution d'un aquifère dans les sables), son épaisseur (intensité dynamique en fonction de seuils d'épaisseur minimale ou maximale), l'évolution de son recul (notion de front de karstification) et son incision (organisation spatiale du liseré karstique en lignes rectilignes, en festons, anneaux, ou vallons).

Lorsque la couverture n'est pas karstifiable, comme les argiles calloviennes de la Wöevre septentrionale, la gaize et l'argile albo-cénomanienne d'Argonne ou les argiles du Keuper dans le Wardnt, un seul front de karstification apparaît avec deux modes d'introduction des eaux qui déterminent un système karstique binaire : une infiltration lente et diffuse dans les calcaires peu fissurés et une infiltration localisée et rapide par des gouffres en limite de couverture.

31 Cette couverture, lorsqu'elle comprend un horizon karstifié, détermine un système karstique ternaire, juxtaposé ou étagé, à l'image des karsts du Barrois (Jaillet, 2000), du Haut-Pays et de la Montagne de Reims. Les couples de couches karstifiées sont respectivement, Hauterivien/ Portlandien, ex Séquanien/ex Argovo-Rauracien et Paléocène Thanétien/Campanien. Pour ces systèmes, les exutoires du karst du niveau supérieur alimentent un réseau hydrographique qui se perd dans la zone d'infiltration du niveau inférieur. Les trois modes d'introduction des eaux par pertes diffuses, pertes localisées et par drainance de la couverture sont ici étagés.

L'endokarst du niveau calcaire soutire par « suffosion $»^{3}$ les matériaux de la couverture affectée alors par des phénomènes karstiques de surface. Dans le cas de la Montagne de Reims, les argiles à meulières (provenant des coulées), ainsi que les sables grésifiés thanétiens, déstructurés par dissolution, présents dans les réseaux souterrains, témoignent de ce processus.

Le contexte de forêt permet d'appeler le karst étudié « karst vert " ${ }^{4}$ à l'image des karsts lorrains. Le nivellement systématique des formes de surface par les travaux agricoles sur terrains cultivés, l'organisation spatiale différente des parcelles agricoles et forestières expliquent la présence d'exokarst uniquement sous forêt.

34 Ces caractéristiques générales font du karst de la Montagne de Reims un karst typique de l'est de la France. Cependant certaines spécificités montrent l'originalité de ce karst.

\section{B. Spécificités du karst de la Montagne de Reims}

Le contexte morphostructural fait du karst de la Montagne de Reims un karst original. En effet, alors que la majeure partie des sites karstifiés sont des revers de côte en limite de recouvrement (Barrois, Wöevre septentrionale, Haut-Pays, Pays-Haut), le karst de la Montagne de Reims est le seul à se situer en position de front de côte et sur un replat structural, ce qui limite considérablement son emprise spatiale. 

d'équipement hydrométrique et d'autre part par la capacité tampon du matériau crayeux, qui lisse considérablement le comportement hydrologique des sources de la craie. Ce karst mériterait un suivi hydrométrique et hydrochimique sur au moins une année hydrologique complète afin de mieux appréhender le comportement hydrodynamique des systèmes karstiques. Des opérations de traçage permettraient de connaître leurs bassins-versants d'alimentation et les modalités d'écoulement.

La nature lithologique des deux niveaux karstifiés, le sable grésifié thanétien et la craie campanienne, est également une originalité par rapport aux karsts lorrains. Le karst de la Montagne de Reims est donc le seul karst de l'est de la France en milieu crayeux sous « caprock sablo-gréseux $»^{5}$.

La discontinuité des formations géologiques paléocènes et surtout thanétiennes, inhérente aux conditions de sédimentation, détermine un liseré karstique particulièrement discontinu et surtout très localisé (éperon oriental de la côte d'Ile-deFrance).

L'endokarst de l'aquifère de la craie et ses principales exsurgences (Fontaine de Trépail, Fontaine de Verzy) en Montagne de Reims sont également originaux car il sont perchés d'une quarantaine de mètres au-dessus de la surface piézométrique de la nappe.

\section{Conclusion et perspectives}

Le karst de la Montagne de Reims est, à plusieurs titres, un karst typique des régions de cuestas de l'est de la France. En effet, c'est un karst de contact lithostratigraphique organisé en front de karstification situé entre deux couches de perméabilité différente. Ce front évolue dans l'espace en fonction de l'état de la couverture. C'est également un karst « vert » développé sous couvert forestier (Parc Naturel Régional de la Montagne de Reims). Il est étagé, c'est-à-dire caractérisé par une double karstification, à la fois des sables grésifiés du Thanétien et de la craie du Campanien sous-jacente. inhérent aux modalités de la sédimentation paléocène, la nature des roches karstifiées (sables grésifiés et craie) et par sa dynamique générale. Cette dernière est caractérisée par une interaction entre la dynamique de versant, représentée par les mouvements de terrain de type rotationnel, et par une dynamique karstique, responsable d'une certaine immunité des replats structuraux.

Cependant ce karst est encore méconnu, surtout dans son fonctionnement hydrogéologique. Cette méconnaissance s'explique d'une part par l'absence totale Ces voies de recherche intéressent la protection des ressources en eau, la genèse et l'évolution des reliefs ainsi que l'incision du réseau hydrographique. En effet, le karst de la Montagne de Reims alimente directement en eau certaines communes du front de côte (captage de Trépail) mais participe également à l'alimentation de la nappe de la craie soumise à une pression anthropique grandissante. L'étude de la genèse et de l'évolution de ce karst participerait également à la réflexion sur la réorganisation du réseau hydrographique et sur le dégagement des reliefs (recul de la couverture tertiaire et du front de côte) au cours du Quaternaire. 


\section{BIBLIOGRAPHIE}

Chauvin J. (1985). - Les cavités du sable du massif de la Montagne de Reims. Spélunca, 19, pp. 39-41.

Chauvin J. (1986). - L'écosystème souterrain du massif de la Montagne de Reims et quelques peuplements phréatiques et hypogés environnants. Thèse de doctorat, faculté des Sciences de Reims, 163 p.

Choppy J. (1985). - Dictionnaire de spéléologie physique et karstologie. Synthèses karstiques, 150 p.

Devos A. (1991). - Ressources et qualités des eaux d'un bassin minier et sidérurgique : la Fensch (Lorraine), Mosella t. XVIII, pp. 239-262.

Devos A. (1996). - Hydrologie et aménagements hydrauliques de la Saulx et de l'Ornain (Lorraine), Septentrion, $443 \mathrm{p}$.

Dumont R. (1959). - La spéléologie dans la montagne de Saint-Basle de Verzy. Bull. Soc. Et. Sci. Nat. de Reims, 42, pp. 7-9.

Duprat M. (1997). - Modèle tectono-sédimentaire des dépôts paléogènes dans le nord-est du bassin de Paris : conséquences sur la géométrie du toit de la craie. Ann. Soc. Géol. Du Nord. T. 5 (2e série), pp. 269-287.

Durup de Baleine A. (1989). - Le karst de la forêt de Hesse. Etude géomorphologique d'un karst vert en Lorraine occidentale. Mémoire de maîtrise de géographie. Université de Nancy II, 286 p.

Durup de Baleine A. (1991). — La Meuse karstique. Le karst de la forêt de Hesse. Connaissance de la Meuse, 19, Bar-le-Duc, 4 p.

Frischmann E. (1997). - Le Muschelkalk supérieur de la périphérie du bassin houiller : problèmes de karstification - aménagement et gestion des ressources. Maîtrise de Géographie, Université de Metz, $111 \mathrm{p}$.

Gamez P. (1995). - Hydrologie et karstologie du bassin du Loison (Wöevre septentrionale Lorraine). Mosella, Tome XXI, $453 \mathrm{p}$.

Guérin H. (1959). - Exploration de la rivière souterraine de Verzy ; Bull. Soc. Et. Sci. Nat. de Reims, 42, pp. 3-7.

Guérin H. (1985). — L'exploitation des meulières en Montagne de Reims de 1792 à 1918, Mémoires de la Société d'agriculture, commerce, sciences et arts du département de la Marne, Tome C, pp. 159-177.

Jaillet S. (2000). - Un karst couvert de bas plateau : le Barrois - structure - fonctionnement - évolution. Thèse de doctorat, Université de Bordeaux 3, 543 p.

Jaillet S., Gamez P. (1995). - Observations morphologiques sur le géosystèmes karstique du Ruptdu-Puits (Lorraine/ Champagne, France). Karstologia, 26, FFS, AFK, Bordeaux, pp. 27-38.

Joly F. (1997). - Glossaire de géomorphologie, base de données sémiologiques pour la cartographie, A. Colin, $325 \mathrm{p}$

Lejeune O. (2000). - Relation entre karst et mouvement de terrain sur le flanc septentrional de la Montagne de Reims ; Mosella, tome XXV, n 1-2, pp. 115-127.

Laurain M. (1980). - Données structurales sur la feuille de Reims 1/50 000e à partir d'observations cartographiques, stratographiques et morphologiques. Bull. Inf. Géol. Bass. Paris, 4, pp. 55-59. 
Laurain M. (1981). - Notice et carte géologique de la feuille de Reims n 132 au 1/50 000, B.R.G.M., 34 p.

Marre A. (1987). - Le mouvement de terrain de Rilly-la-Montagne du 23 août 1986 : naissance et évolution; Travaux de l'Institut de Géographie de Reims, n 69-72, Reims, pp. 95-111.

Nicod J. (1992). - Les karsts sous couverture (sableuse, argileuse et/ou détritique) en France, d'après des travaux récents. Historia, 20, pp. 165-185.

Nicod J. (1994). - Plateaux karstiques sous couverture en France ; Ann. Géographie, 576, pp. 170-194.

Nicod J. (1995). - Carte géomorphologique des karsts de France - Notice d'une maquette au 1/3 000000 environ. Karstologia, $\mathrm{n}^{\circ}$ 25-2, pp. 21-34.

Quinif Y. (1998). - Dissipation d'énergie et adaptabilité dans les systèmes karstiques, Karstologia, $\mathrm{n}^{\circ} 31, \mathrm{pp} .1-11$.

Rodet J. (1985). - Karst de la craie de la cuesta de l'Ile-de- France : la Montagne de Reims orientale. Spélunca mémoires, ${ }^{\circ}$ 14, p. 75-77.

Rodet J. (1992). - La craie et ses karsts. Thèse de Doctorat d'état. Université Aix-Marseille II, 560 p. Rodet J. (1997). - Typologie des karsts dans la craie du Bassin de Paris. Ann. Soc. Géol. Du Nord. T.5 (2e série), pp. 351-359.

\section{NOTES}

1. Compresse humide: formation superficielle ou géologique perméable ou semi-perméable alimentant par drainance les horizons sous-jacents.

2. Versant réglé-glacis : «Versant périglaciaire rectiligne, en pente forte $\left(30-35^{\circ}\right)$, régularisé par gélifraction et dénudé par gravité » (Joly, 1997). Il constitue la partie sommitale du versant alors que la partie basale forme un glacis, "surface plane de bas de versant en pente faible $\left(10^{\circ}\right)$ » (Joly, 1997).

3. Suffosion: "soutirage en fonction d'un écoulement hypodermique ou souterrain peu profond » (Joly, 1997).

4. Karst vert : autre nom du «karst forestier» (Choppy, 1985), c'est un karst sous couverture forestière.

5. Caprock sablo-gréseux: couverture rocheuse composée de couches sableuses et argileuses localement ou intégralement consolidées.

\section{RÉSUMÉS}

La Montagne de Reims est affectée par un karst de contact lithostratigraphique à l'image des autres karsts de l'est du Bassin parisien. Comme eux, il est également étagé et sous couverture forestière. Cependant, il présente certaines spécificités : c'est un karst de front de côte qui s'inscrit en milieu crayeux et se trouve perché au-dessus de la nappe de la craie. Il est également accompagné par des mouvements de terrain de type rotationnel qui conditionnent son évolution. 
The "Montagne de Reims" karst is a karst of lithostratigraphic contact as are others of the eastern Paris Basin. Like them, this karst also occurs at different levels beneath forest cover. However, it has certain specialities: it is a karst of the scarp face developed in Middle Chalk and is perched above the Chalk water table. It is also affected by the movements of rotational landslides which condition its evolution.

Der Karst der Montagne de Reims ist ein vom lithostratigraphischer Kontaktkarst wie die anderen Karstgebiete des östlichen Pariser Beckens. Wie sie ist er gestuft und unter Waldbedeckung. Doch zeigt er gewisse Besonderheiten. Es ist ein Karst der Stufenstirn im Bereich der Kreide über dem Grundwasser-spiegel. Es haben sich aus Rotationsbewegungen im Boden ausgewirkt, welche die Entwicklung des Karsts bedingen.

\section{INDEX}

Keywords : Chalk, cuesta, karst, landslide, Montagne de Reims

Schlüsselwörter : Cuesta, Karst, Kreide, Montagne de Reims, Rotationsbodenbewe-gungen

Mots-clés : craie, cuesta, karst, Montagne de Reims, mouvement de terrain

\section{AUTEURS}

OLIVIER LEJEUNE

GAGE-GREURCA, Université de Reims Champagne-Ardenne, 57, rue Pierre-Taittinger 51096 Reims Cedex, olivier.lejeune3@libertysurf.fr

\section{ALAIN DEVOS}

GAGE-GREURCA, Université de Reims Champagne-Ardenne, 57, rue Pierre-Taittinger 51096 Reims

Cedex, alain.devos@univ-reims.fr 